COMMUNICATIONS IN

ANALYSIS AND GEOMETRY

Volume 12, Number 4, 821-836, 2004

\title{
Minimal Planes in Hyperbolic Space
}

\author{
BARIS COSKUnuzer
}

In this paper we show a generic finiteness result for least area planes in $\mathbb{H}^{3}$. Moreover, we prove that the space of minimal immersions of disk into $\mathbb{H}^{3}$ is a submanifold of product bundle over a space of immersions of circle into $S_{\infty}^{2}\left(\mathbb{H}^{3}\right)$ and the bundle projection map is when restricted to this submanifold is Fredholm of index zero. Using this, we also show that the space of minimal planes with smooth boundary curve at infinity is a manifold.

\section{Introduction.}

The asymptotic Plateau problem in $\mathbb{H}^{3}$ has been solved by Anderson in [A1], by using geometric measure theory methods. In this paper, we will consider this problem, by using geometric analysis, and global analysis methods. Indeed, in a sense, we will try to translate Anderson's results to these two fields to get another perspective. We do not have any extra results about the asymptotic Plateau problem, but we got a very nice global picture of space of minimal planes, and by using this, we will prove generic finiteness results.

A minimal plane $\mathrm{P}$ is a plane such that the mean curvature $\mathrm{H}$ is 0 at every point of x, i.e. $\forall x \in P, H(x)=0$. By Eells and Sampson's results in [ES], for any minimal plane $\Sigma$ in $\mathbb{H}^{3}$, there is a conformal harmonic parametrization map $u: D^{2} \rightarrow \mathbb{H}^{3}$ with $u\left(D^{2}\right)=\Sigma$. This establishes the starting point for our approach.

In this paper, we will only consider minimal planes with $C^{3, \mu}$ regular asymptotic boundary. We will consider this space as the space of minimal immersions of $D^{2}$ into $\mathbb{H}^{3}$, i.e. conformal harmonic maps from disk to hyperbolic space. Then, one can think this space as a subspace of harmonic maps from disk to hyperbolic space, and the minimal immersions in this space are the conformal ones. As a result, we study the minimal maps as a subspace of harmonic maps.

The space of harmonic maps from disk to hyperbolic space has very nice features by Li and Tam's results. With some regularity conditions, the space of harmonic maps from $D^{2}$ to $\mathbb{H}^{3}$ can be identified with their induced 
boundary parametrization $\partial D^{2}$ to $S_{\infty}^{2}\left(\mathbb{H}^{3}\right)$, by existence and uniqueness of harmonic extensions in [LT1] and [LT2]. So, the space of minimal maps can be considered as the subspace of immersions of $S^{1}$ into $S_{\infty}^{2}\left(\mathbb{H}^{3}\right)$.

Next, we will show that the space of minimal maps is a submanifold of the space of immersions of $S^{1}$ into $S_{\infty}^{2}\left(\mathbb{H}^{3}\right)$. Also, by factoring out the parametrizations, we prove that the space of minimal planes is a manifold, too.

Moreover, we will prove that the boundary restriction map from the space of minimal immersions into the immersions of $S^{1}$ into $S_{\infty}^{2}\left(\mathbb{H}^{3}\right)$ is Fredholm map of index 0 . In other words, for a generic $C^{3, \mu}$ Jordan curve $\Gamma \subset S_{\infty}^{2}\left(\mathbb{H}^{3}\right)$, the space of minimal planes spanning this curve, $M(\Gamma)$, is a collection of isolated points.

Theorem A: $M=\left\{(\alpha, u) \in C^{3, \mu}\left(S^{1}, S^{2}\right) \times C^{3}\left(S^{1}, S^{1}\right) \mid \widetilde{\alpha \circ u}\left(D^{2}\right)\right.$ is minimal $\}$ is a submanifold of the product bundle $C^{3, \mu}\left(S^{1}, S^{2}\right) \times$ $C^{3}\left(S^{1}, S^{1}\right)$, and the bundle projection map restricted to $M,\left.\Pi_{1}\right|_{M}$, is Fredholm of index 0 .

Theorem B: $M_{0}=\left\{\Sigma \subset \mathbb{H}^{3} \mid \Sigma\right.$ minimal, $\partial_{\infty} \Sigma \subset S_{\infty}^{2}\left(\mathbb{H}^{3}\right)$ is class of $\left.C^{3}\right\}$ the space of immersed minimal planes is a manifold.

The other main result is the generic finiteness of least area planes spanning a fixed curve $\Gamma \subset S_{\infty}^{2}\left(\mathbb{H}^{3}\right)$. By Theorem $\mathrm{A}$, for a generic curve $\Gamma$, the set of least area planes spanning it, $M(\Gamma)$, is a collection of isolated points. So all we need to show is that $M(\Gamma)$ is compact. We will accomplish this by showing that this set is a subset of a compact set by using boundary regularity results on minimal planes in hyperbolic space by Hardt and Lin.

Theorem C: Let $\Gamma \subset S_{\infty}^{2}\left(\mathbb{H}^{3}\right)$ be a $C^{3, \mu}$ generic Jordan curve. Then there are finitely many least area planes spanning this curve.

The organization of the paper is as follows: In Section 2, we will give some preliminary definitions and theorems, which will be used for the rest of the paper. In Section 3, we study the structure of the space of minimal planes, and show that it is a manifold. In Section 4, we will prove the generic finiteness result. Finally, in Section 5, we will have some concluding remarks.

\subsection{Acknowledgements.}

I am very grateful to my advisor David Gabai for his continuous encouragement and advice. I would like to thank Simon Brendle, Alice Chang, Paul Yang, Fengbo Hang and Fang-Hua Lin for very helpful conversations. 


\section{Preliminaries.}

In this section, we will parametrize the space of minimal immersions of disk into hyperbolic space.

Lemma 2.1. [ES](Minimal immersions and harmonic maps) If $\varphi$ is an isometric immersion, then $\varphi$ is minimal if and only if it is harmonic.

Theorem 2.2. Let $\Sigma$ be a plane in $\mathbb{H}^{3}$. Then $\Sigma$ is minimal if and only if there is a conformal harmonic map $u: D^{2} \rightarrow \mathbb{H}^{3}$ with $u\left(D^{2}\right)=\Sigma$.

Proof. Let $\Sigma$ be a minimal plane in $\mathbb{H}^{3}$. Let $u: D^{2} \rightarrow \mathbb{H}^{3}$ be an immersion with $u\left(D^{2}\right)=\Sigma$. Let $g$ be the induced metric on $D^{2}$. Then $u:\left(D^{2}, g\right) \rightarrow$ $\Sigma \subset \mathbb{H}^{3}$ is an isometric immersion. By Lemma 2.1 , we conclude that $\mathrm{u}$ is harmonic with respect to the induced metric $g$.

By uniformization theorem, $\left(D^{2}, g\right)$ is conformally equivalent to $\mathbb{C}$ or Euclidean disk $\left(D^{2}, g_{0}\right)$. But, since $\mathrm{g}$ is induced by $\Sigma$, mean curvature 0 plane, in $\mathbb{H}^{3}$, constant sectional curvature -1 space, then $\left(D^{2}, g\right)$ is conformally equivalent to $\left(D^{2}, g_{0}\right)$. So, there exist a conformal map $\phi:\left(D^{2}, g_{0}\right) \rightarrow\left(D^{2}, g\right)$. Since harmonicity respects conformality, $u \circ \phi:\left(D^{2}, g_{0}\right) \rightarrow \Sigma$ is harmonic. Then, there exist a conformal harmonic map $v: D^{2} \rightarrow \Sigma$.

The reverse direction is trivial, as by definition, for any conformal harmonic map, the mean curvature is 0 at every point in the image.

So, by using this theorem, we can consider minimal planes as conformal harmonic maps. Now, let's focus on harmonic maps.

Lemma 2.3. [LT1][LT2](Harmonic extensions) If $\gamma: S_{\infty}^{1}\left(\mathbb{H}^{2}\right) \rightarrow S_{\infty}^{2}\left(\mathbb{H}^{3}\right)$ a $C^{1}$ immersion, then there exist unique $C^{1}$-regular harmonic extension map $\overline{\mathbb{H}^{2}} \rightarrow \overline{\mathbb{H}^{3}}$.

Lemma 2.4. $[L][\mathrm{To}]$ (Boundary regularity of minimal planes) If $\Gamma \subset$ $S_{\infty}^{2}\left(\mathbb{H}^{3}\right)$ is a $C^{1}$ curve, and $\Sigma$ is minimal plane with $\partial_{\infty} \Sigma=\Gamma$, then $\Sigma \cup \Gamma$ is $C^{1}$, too.

Theorem 2.5. Let $M=\left\{u: D^{2} \rightarrow \mathbb{H}^{3} \mid u\right.$ minimal immersion and $\left.u\right|_{\partial D^{2}}$ is $\left.C^{1}\right\}$, and let $A=\left\{\alpha: S^{1} \rightarrow S^{2} \mid \alpha\right.$ immersion $\}$. Then $\beta: M \rightarrow A$ boundary restriction map is one to one. 
Proof. Let $\mathrm{u}$ be a minimal immersion with $\left.u\right|_{\partial D^{2}}$ is $C^{1}$. Then $\alpha:=\left.u\right|_{\partial D^{2}}$ is in A, the image of $\mathrm{u}$ under the boundary restriction map. This map is 1-1, since by Lemma 2.4, the image of $\mathrm{u}$ is $C^{1}$ and by theorem 2.3, there exist unique $C^{1}$ harmonic extension of $\alpha$, which must be $u$. So, $\beta$ embeds $M$ into A.

So, we can identify $\mathrm{M}$ with $\beta(M) \subset A$, and we can consider the space of minimal immersions $\mathrm{M}$ as a subspace of space of immersions of $S^{1}$ into $S^{2}$. In the following sections, we will use this identification.

\section{The Space of Minimal Planes.}

In this section, we will show that the space of minimal immersion is a manifold, and boundary restriction map is Fredholm of index 0. Basically, we will adapt the techniques of Tomi and Tromba in [TT] and [To].

We will start by defining the following spaces: Let $\mu \geq 0$.

$A=\left\{\alpha \in C^{3, \mu}\left(S^{1}, S^{2}\right) \mid \alpha\right.$ immersion $\}$

$D=\left\{u \in C^{3}\left(S^{1}, S^{1}\right) \mid u\right.$ diffeomorphism and satisfies three point condition, i.e. $\left.u\left(e^{\frac{2}{3} k \pi i}\right)=e^{\frac{2}{3} k \pi i}, k=1,2,3\right\}$

$M=\left\{f: D^{2} \rightarrow \mathbb{H}^{3} \mid f\left(D^{2}\right)\right.$ minimal and $\left.\left.f\right|_{\partial D^{2}} \in A\right\}$

$N=A \times D$

Here, A is open subset of $C^{3, \mu}\left(S^{1}, S^{2}\right)$, D is a smooth manifold, and so is $\mathrm{N}$.

Define the conformality operator $k: N \rightarrow C^{2}\left(S^{1}, \mathbb{R}\right)$ such that

$$
k(\alpha, u)=\frac{\partial}{\partial r}(\alpha \circ u) \cdot \frac{\partial}{\partial \theta}(\alpha \circ u)
$$

Here, $r, \theta$ represents polar coordinates on $D^{2}$ and functions $\alpha \circ u$ identified with their harmonic extensions, $\widetilde{\alpha \circ u}$. So, conformality operator is dot product of partial derivatives of $\alpha \circ u$.

Lemma 3.1. $\operatorname{ker}(k) \subset N$ consists of conformal harmonic maps, i.e. $k(\alpha, u)=0$ if and only if the harmonic extension $\widetilde{\alpha \circ u}$ is conformal.

Proof. Let

$$
\delta=(\cos \theta-i \sin \theta)\left(\frac{\partial}{\partial r}-\frac{i}{r} \frac{\partial}{\partial \theta}\right)
$$

be the complex differential. Then

$$
k(\alpha, u)=-\operatorname{Im}\left(z^{2} \delta(\alpha \circ u) \cdot \delta(\alpha \circ u)\right)
$$


But $\delta(\widetilde{\alpha \circ u}) \cdot \delta(\widetilde{\alpha \circ u})$ is the Hopf differential of $\widetilde{\alpha \circ u}$,

$$
Q(\widetilde{\alpha \circ u})=\left(|\widetilde{\alpha \circ u}|_{x}^{2}-|\widetilde{\alpha \circ u}|_{y}^{2}\right)-2 i\left((\widetilde{\alpha \circ u})_{x} \cdot(\widetilde{\alpha \circ u})_{y}\right)
$$

The Hopf differential, $Q$, is holomorphic for harmonic maps, [ER]. Then, $Q(\widetilde{\alpha \circ u})=0$ if and only if $\widetilde{\alpha \circ u}$ is conformal. Since $\operatorname{Im}\left(z^{2} \delta(\widetilde{\alpha \circ u}) \cdot \delta(\widetilde{\alpha \circ u})\right)$ is harmonic function, then $k(\alpha, u)=0$ implies $Q(\widetilde{\alpha \circ u})=0$, which means $\widetilde{\alpha \circ u}$ is conformal.

To understand the structure of $\operatorname{ker}(k)$, we will study the derivative of $k$ :

$$
\begin{gathered}
D_{\alpha} k(\alpha, u)\langle\beta\rangle=\frac{\partial}{\partial r}(\beta \circ u) \cdot \frac{\partial}{\partial \theta}(\alpha \circ u)+\frac{\partial}{\partial r}(\alpha \circ u) \cdot \frac{\partial}{\partial \theta}(\beta \circ u) \\
D_{u} k(\alpha, u)\langle v\rangle=\frac{\partial}{\partial r}[(D \alpha) \circ u\langle v\rangle] \cdot \frac{\partial}{\partial \theta}(\alpha \circ u)+\frac{\partial}{\partial r}(\alpha \circ u) \cdot \frac{\partial}{\partial \theta}[(D \alpha) \circ u\langle v\rangle]
\end{gathered}
$$

Lemma 3.2. $[A D N]$ Let $a_{1}, a_{2}, a_{3}, b \in C^{2}\left(S^{1}, \mathbb{R}^{3}\right)$ such that for every $p \in$ $S^{1}$, the vectors $a_{1}(p), a_{2}(p)$, and $a_{3}(p)$ are linearly independent. Then the following system is an elliptic boundary value system.

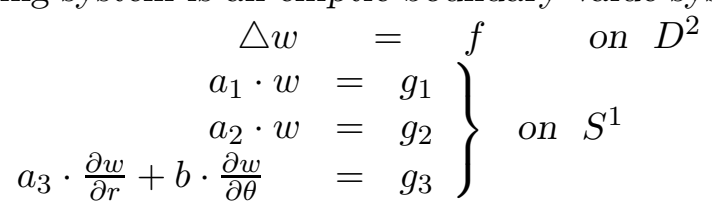

Lemma 3.3. [BT] Let $F, X_{1}, X_{2}$ be Banach spaces and let $A_{k}: F \rightarrow X_{k}$ with $k=1,2$ be linear operators. Let $A:=\left(A_{1}, A_{2}\right): F \rightarrow X_{1} \times X_{2}$ be Fredholm operator. Then $L:=\left.A_{1}\right|_{A_{2}^{-1}(0)}$ is also a Fredholm operator.

Corollary 3.4. The map $w \rightarrow a_{3} \cdot \frac{\partial w}{\partial r}+b \cdot \frac{\partial w}{\partial \theta}$ from $\left\{w \in C^{3, \mu}\left(S^{1}, \mathbb{R}^{3}\right) \mid \triangle_{\mathbb{H}} w=\right.$ $\left.0, a_{1} \cdot w=a_{2} \cdot w=0\right\}$ into $C^{2}\left(S^{1}, \mathbb{R}\right)$ is a Fredholm operator.

Proof. Since the system in Lemma 3.2 is elliptic boundary value system, then the operator

$$
w \rightarrow\left(\triangle w, a_{1} \cdot w, a_{2} \cdot w, a_{3} \cdot \frac{\partial w}{\partial r}+b \cdot \frac{\partial w}{\partial \theta}\right)
$$

for the suitable spaces is Fredholm, [LRW]. Then by Lemma 3.3, the map

$$
w \rightarrow a_{3} \cdot \frac{\partial w}{\partial r}+b \cdot \frac{\partial w}{\partial \theta}
$$


from $\left\{w \in C^{3}\left(S^{1}, \mathbb{R}^{3}\right) \mid \triangle w=0, a_{1} \cdot w=a_{2} \cdot w=0\right\}$ into $C^{2}\left(S^{1}, \mathbb{R}\right)$ is also Fredholm. Since $\triangle w=0$ condition is essentially same in the interior with $\triangle_{\mathbb{H}} w=0$ condition, then the result follows.

Theorem 3.5. $D_{u} k(\alpha, u): T_{u} D \rightarrow C^{2}\left(S^{1}, \mathbb{R}\right)$ is Fredholm where $(\alpha, u) \in$ $N$.

Proof. For $v \in T_{u} D$,

$$
D_{u} k(\alpha, u)\langle v\rangle=\frac{\partial}{\partial r}[(D \alpha) \circ u\langle v\rangle] \cdot \frac{\partial}{\partial \theta}(\alpha \circ u)+\frac{\partial}{\partial r}(\alpha \circ u) \cdot \frac{\partial}{\partial \theta}[(D \alpha) \circ u\langle v\rangle]
$$

Let $N^{\alpha}=\{\alpha \circ u \mid u \in D\}$. Then by using the isomorphism $T_{u} D \rightarrow T_{\alpha \circ u} N^{\alpha}$ with $v \rightarrow(D \alpha) \circ u\langle v\rangle$, we can simplify $D_{u} k(\alpha, u)$ with $w=(D \alpha) \circ u\langle v\rangle$. By abuse of notation, we will have $D_{u} k(\alpha, u): T_{\alpha \circ u} N^{\alpha} \rightarrow C^{2}\left(S^{1}, \mathbb{R}\right)$

$$
D_{u} k(\alpha, u)\langle w\rangle=a_{3} \cdot \frac{\partial w}{\partial r}+b \cdot \frac{\partial w}{\partial \theta}
$$

where $a_{3}=\frac{\partial}{\partial \theta}(\alpha \circ u)$ and $b=\frac{\partial}{\partial r}(\alpha \circ u)$.

Then, we can find vector functions $a_{1}, a_{2} \in C^{2}\left(S^{1}, \mathbb{R}^{3}\right)$ such that $\left\{a_{1}, a_{2}, a_{3}\right\}$ form an orthogonal triple and

$$
T_{\alpha \circ u} N^{\alpha}=\left\{w \in C^{3}\left(S^{1}, \mathbb{R}^{3}\right) \mid \triangle_{\mathbb{H}} w=0, a_{1} \cdot w=a_{2} \cdot w=0\right\}
$$

by the definition of $a_{3}$. By Lemma 3.2 and Lemma $3.3, D_{u} k$ is a Fredholm operator.

Theorem 3.6. The Fredholm index of $D_{u} k(\alpha, u)$ is 0 for $(\alpha, u) \in N$.

Proof. We will prove this theorem in 3 steps.

Claim 1: Let

$$
Z=\left\{h \in C^{2}\left(S^{1}, \mathbb{R}\right) \mid \int_{S^{1}} h d \theta=\int_{S^{1}} h \cos \theta d \theta=\int_{S^{1}} h \sin \theta d \theta=0\right\}
$$

be the subspace of $C^{2}\left(S^{1}, \mathbb{R}\right)$. Then the image of $k$ is contained in Z, i.e. $k(A \times D) \subset Z \subset C^{2}\left(S^{1}, \mathbb{R}\right)$.

Proof. By above, $k(\alpha \circ u)=-\operatorname{Im}\left(\left.z^{2} Q(\widetilde{\alpha \circ u})\right|_{\partial D^{2}}\right)$ Since $Q$ is holomorphic, the result follows by Cauchy's theorem.

Claim 2: $D_{u} k(i d, i d): T_{i d} D \rightarrow Z$ is an isomorphism, where $i d$ is the identity of $S^{1}$. 
Proof. First, we will show that $D_{u} k$ is injective. If we identify the elements of $D$ with their harmonic extensions, then the elements of $T_{i d} D$ will be harmonic functions from $D^{2}$ to $\mathbb{R}^{2}$. so, we can think of them as complex valued functions. Let $v \in T_{i d} D$.

$$
\begin{aligned}
D_{u} k(i d, i d)\langle v\rangle & =\frac{\partial v}{\partial r} \cdot \frac{\partial i d}{\partial \theta}+\frac{\partial i d}{\partial r} \cdot \frac{\partial v}{\partial \theta} \\
& =\operatorname{Re}\left(\frac{\partial v}{\partial r} \cdot \frac{\partial i d}{\partial \theta}+\frac{\partial v}{\partial \theta} \cdot \frac{\partial i d}{\partial r}\right) \\
& =\operatorname{Re}((z \delta v+\bar{z} \bar{\delta} v)(-i \bar{z})+(i z \delta v-i \bar{z} \bar{\delta} v) \bar{z}) \\
& =\operatorname{Re}\left(-i \bar{z}^{2} \bar{\delta} v\right)=\operatorname{Re}\left(i z^{2} \delta \bar{v}\right)
\end{aligned}
$$

Since $\mathrm{v}$ is harmonic, $\delta \bar{v}$ is holomorphic. So, $\operatorname{Re}\left(i z^{2} \delta \bar{v}\right)=0$ implies $i z^{2} \delta \bar{v}=$ 0 and $\mathrm{v}$ is itself holomorphic. Now, since for any $u \in D, u\left(S^{1}\right)=S^{1}$ and $v \in T_{i d} D, \mathrm{v}$ is tangential to $S^{1}$, then the function

$$
w=\frac{v}{i z}
$$

is real valued on $S^{1}$. By reflection principle, we can extend w to a meromorphic function $\hat{w}$ on $\overline{\mathbb{C}}$ with simple poles at 0 and $\infty$. Then by elementary complex analysis,

$$
w(z)=a+b z+\frac{\bar{b}}{z}
$$

and

$$
v(z)=i\left(\bar{b}+a z+b z^{2}\right)
$$

where $a \in \mathbb{R}$ and $b \in \mathbb{C}$. Then, by the three point condition, v must be 0 . So, $D_{u} k(i d, i d)$ is injective.

Now, we will show surjectivity. Let $h \in Z$. Then there exist a holomorphic function $g$ with boundary values of class $C^{2}$ such that $h=\operatorname{Re} g$, $g(0)=g^{\prime}(0)=0$. So, $g$ can be written as $g=i z^{2} f$ where $f$ is a holomorphic function with boundary values of class $C^{2}$. We claim that there is a $v \in T_{i d} D$, such that $\operatorname{Re}\left(i z^{2} \delta \bar{v}\right)=h$.

Set

$$
v=\bar{F}+H
$$

where $F$ and $H$ are holomorphic and $F^{\prime}=f$. Obviously, for such $v$, $\operatorname{Re}\left(i z^{2} \delta \bar{v}\right)=h$ holds. All we need to do is to find a suitable $H$ such that $v=\bar{F}+H \in T_{i d} D . v$ is tangential to $S^{1}$ if and only if $\operatorname{Re}(\bar{z} v)=0$ on $S^{1}$. So, we need 


$$
\operatorname{Re}(\bar{z} H)=-\operatorname{Re}(z F) \quad \text { or } \quad \operatorname{Re}\left(\frac{H}{z}\right)=-\operatorname{Re}(z F)
$$

Then, if we put

$$
H=-z^{2} F+i\left(\bar{b}+a z+b z^{2}\right)
$$

where $a \in \mathbb{R}$ and $b \in \mathbb{C}$ are chosen in such a way that $\mathrm{v}$ satisfies the three point condition. So, $D_{u} k(i d, i d)$ is surjective.

Claim 3: For $(\alpha, u) \in N$, the Fredholm index of $D_{u} k(\alpha, u)$ is 0 .

Proof. Consider the map $\Phi: N \rightarrow L\left(E_{1}, E_{2}\right)$ from $N$ into bounded linear operators, with $\Phi(\alpha, u)=D_{u} k(\alpha, u)$ where $E_{1}=T_{i d} D$ and $E_{2}=C^{2}\left(S^{1}, \mathbb{R}\right)$. Here we identified $T_{u} D$ with $T_{i d} D$ for any $u \in D$, with abuse of notation. Then $\Phi(N) \subset F\left(E_{1}, E_{2}\right) \subset L\left(E_{1}, E_{2}\right)$ where $F\left(E_{1}, E_{2}\right)$ represents Fredholm operators. By $[\mathrm{Sm}]$, index is continuous on Fredholm operators. Since for any $(\alpha, u) \in N$, there exist a path in $N$ connecting $(\alpha, u)$ to $(i d, i d)$, by the existence of homotopies in the corresponding spaces. Then, continuity of index implies ind $\left(D_{u} k(\alpha, u)\right)=\operatorname{ind}\left(D_{u} k(i d, i d)\right)=0$.

Let $M=\{(\alpha, u) \in N \mid k(\alpha, u)=0, \delta(\widetilde{\alpha \circ u}) \neq 0\}$ represents the space of minimal immersions of $D^{2}$ into $\mathbb{H}^{3}$. Here, for any $(\alpha, u) \in M, \widetilde{\alpha \circ u}: D^{2} \rightarrow$ $\mathbb{H}^{3}$ is minimal immersion.

Theorem 3.7. $M$ is a submanifold of $N$ with tangent space $T_{(\alpha, u)} M=$ $\operatorname{ker}(D k(\alpha, u))$.

Proof. We will prove this theorem by showing that if $(\alpha, u) \in M \subset N$ then $D k(\alpha, u): T_{(\alpha, u)} N \rightarrow Z$ is onto.

$$
D k(\alpha, u)\langle\beta, v\rangle=\frac{\partial w}{\partial r} \cdot \frac{\partial}{\partial \theta}(\alpha \circ u)+\frac{\partial}{\partial r}(\alpha \circ u) \cdot \frac{\partial w}{\partial \theta}
$$

where $w=\beta \circ u+(D \alpha) \circ u\langle v\rangle$.

Consider the simple functional analytic fact: Let $X, Y$ be Banach spaces, $X_{0}$ be a dense subspace of $X$, and $T: X \rightarrow Y$ be linear and continuous. If $T$ is onto and $T\left(X_{0}\right)$ is closed, then $T\left(X_{0}\right)=Y$.

Set $X_{0}=$ Range $\{\beta \rightarrow \beta \circ u\} \times T_{u} D, X=C^{3}\left(S^{1}, T S^{2}\right) \times T_{u} D$, and $Y=Z$. We know Range $(D k(\alpha, u))$ is closed by Theorem 3.5. So, by the above fact, it suffices to solve the equation

$$
\frac{\partial w}{\partial r} \cdot \frac{\partial}{\partial \theta}(\alpha \circ u)+\frac{\partial}{\partial r}(\alpha \circ u) \cdot \frac{\partial w}{\partial \theta}=h
$$


in $X$ for $h \in Z \subset C^{2}\left(S^{1}, \mathbb{R}\right)$.

In complex notation, this equation becomes $\operatorname{Im}\left(z^{2} \delta w \cdot \delta(\alpha \circ u)\right)=\operatorname{Im}\left(z^{2} g\right)$, where $g$ is holomorphic in the unit disc and has boundary values $C^{2}\left(S^{1}\right)$. Let's denote this space by $\Psi$.

Define another space $\Phi=\left\{V: D^{2} \rightarrow \mathbb{R}^{3} \mid \nabla_{\bar{z}} V=0\right.$, and $\left.V\right|_{\partial D^{2}}$ is class of $\left.C^{2}\right\}$ where $\nabla$ is covariant derivative. Recall that $\varphi$ is harmonic map if and only if $\nabla_{\bar{z}} \partial_{z} \varphi=0$.

Then it is sufficient to solve the equation $W \cdot F=g$, where $g \in \Psi$, $F=\delta(\widetilde{\alpha \circ u})$ are given, $F$ does not vanish in the unit disc and $W=\delta \widetilde{w}$ is unknown. Since $\widetilde{\alpha \circ u}, \widetilde{w}$ are harmonic maps, then

$$
\nabla_{\bar{z}} \delta(\widetilde{\alpha \circ u})=\nabla_{\bar{z}} \delta \widetilde{w}=0
$$

so $F, W \in \Phi$.

Let $V_{1}, V_{2} \in \Phi$, then consider

$$
\partial_{\bar{z}}\left(V_{1} \cdot V_{2}\right)=\left(\nabla_{\bar{z}} V_{1} \cdot V_{2}\right)+\left(V_{1} \cdot \nabla_{\bar{z}} V_{2}\right)=0
$$

so $V_{1} \cdot V_{2} \in \Psi$.

We will show that there exist a solution to the equation $W \cdot F=g$ by using the fact that Banach space $\Psi$ is a topological algebra. Define the set $J=\Phi \cdot F$. If the equation is not solvable for some $g \in \Psi$, then $\mathrm{J}$ is an ideal of $\Psi$. Since any ideal is contained in some maximal ideal, we may conclude from the Gelfand-Mazur Theorem that $\mathrm{J}$ is contained in the kernel of some algebra homomorphism $\tau: \Psi \rightarrow \mathbb{C}$.

By using the norm

$$
\|f\|=\left(\sum_{j=0}^{\infty}\left(1+k^{2}\right)\left|a_{k}\right|^{2}\right)^{\frac{1}{2}}
$$

for $f=\sum a_{k} z^{k} \in \Psi$, we get

$$
|\tau(i d)|=\sqrt[n]{\mid \tau\left(i d^{n}\right)} \leq \sqrt[n]{\|\tau\|} \sqrt[n]{\left\|i d^{n}\right\|}
$$

Then,

$$
|\tau(i d)| \leq \limsup _{n \rightarrow \infty} \sqrt[n]{\left\|i d^{n}\right\|}=\lim \sup \left(1+n^{2}\right)^{\frac{1}{2 n}}=1
$$

So, $\xi_{0}=\tau(i d)$ is a point of the unit disc and, for an arbitrary $f=$ $\sum a_{k} z^{k} \in \Phi$, we have

$$
\tau(f)=\sum a_{k} \tau(i d)^{k}=f\left(\xi_{0}\right)
$$


Since $J \subset \operatorname{ker}(\tau)$, for any $W \in \Phi$, we have

$$
0=\tau(W \cdot F)=W\left(\xi_{0}\right) \cdot F\left(\xi_{0}\right)
$$

But this implies $F\left(\xi_{0}\right)=0$, which contradicts to the immersion assumption.

Lemma 3.8. [TT] Let $X_{1}, X_{2}, Y$ be Banach spaces and $L: X_{1} \times X_{2} \rightarrow Y$ be a linear and surjective operator such that $L_{2}:=L(0, \cdot): X_{2} \rightarrow Y$ is Fredholm. Let $\Pi$ denote the projection $X_{1} \times X_{2} \rightarrow X_{1}$ and $M:=\operatorname{ker} L$. Then we have $\operatorname{ker}\left(\left.\Pi\right|_{M}\right)=\operatorname{ker}\left(L_{2}\right)$, and $\operatorname{coker}\left(\left.\Pi\right|_{M}\right)=\operatorname{coker}\left(L_{2}\right)$, i.e. $\left.\Pi\right|_{M}$ is Fredholm and ind $\left(\left.\Pi\right|_{M}\right)=\operatorname{ind}\left(L_{2}\right)$.

Theorem 3.9. The restriction of the projection map $\Pi: A \times D \rightarrow A$ to the submanifold $M,\left.\Pi\right|_{M}$ is onto and $C^{2}$ Fredholm of index 0 .

Proof. $\left.\Pi\right|_{M}$ is onto, since for any $\alpha \in A, \Gamma=\alpha\left(S^{1}\right) \subset S_{\infty}^{2}\left(\mathbb{H}^{3}\right)$ is a $C^{3, \mu}$ curve, and there is a minimal (indeed, least area) plane $\Sigma \subset \mathbb{H}^{3}$ spanning $\Gamma$ by Anderson's results [A1]. Then by Theorem 2.2, there is a conformal harmonic parametrization of $\Sigma$, say $\varphi$. Since there exist $u \in D$ such that $\left.\varphi\right|_{\partial D^{2}}=\alpha \circ u$, then $(\alpha, u) \in M$. This implies $\Pi_{M}$ is onto.

Let $(\alpha, u) \in M$. Then by using the above lemma with, $X_{1}=T_{\alpha} A$, $X_{2}=T_{u} D, Y=Z, L=D k(\alpha, u)$, we get $\operatorname{ind}\left(\left.\Pi\right|_{M}\right)=\operatorname{ind}\left(D_{u} k(\alpha, u)\right)$. Then by Theorem 3.6, the result follows.

So far, we proved that the space of minimal immersions, $M$, is a submanifold of $N$. But, in this space, for a given minimal plane $\Sigma \subset \mathbb{H}^{3}$, there are different corresponding parametrizations. For example, if $\Sigma=(\widetilde{\alpha \circ u})\left(D^{2}\right)$ then $\Sigma$ is represented by $(\alpha, u) \in M$. But, for any $v \in D,\left(\alpha \circ v, v^{-1} \circ u\right) \in M$ represents same minimal plane $\Sigma$.

All results in this section up to now are valid for any $\mu \geq 0$. Now, we will show for $\mu=0$, the space of minimal planes is a manifold. Let $M_{0}=\left\{\Sigma \subset \mathbb{H}^{3} \mid \Sigma\right.$ minimal, $\partial_{\infty} \Sigma \subset S_{\infty}^{2}\left(\mathbb{H}^{3}\right)$ is class of $\left.C^{3}\right\}$ be the space of minimal planes. By above discussion to get $M_{0}$, we have to factor out the group actions from $M$.

Lemma 3.10. $M_{0}=M_{1} / C$, where $M_{1}=M / D$ and $C$ is the space of conformal diffeomorphisms of the unit disk. 
Proof. Consider the $D$ action on $M$. For any $v \in M, \Psi_{v}: M \rightarrow M$ such that $\Psi_{v}(\alpha, u)=\left(\alpha \circ v, v^{-1} \circ u\right)$. Obviously, $D$ action does not change the induced harmonic extension $\widetilde{\alpha \circ u}$ as the composition of $\left(\alpha \circ v \circ v^{-1} \circ u\right)=\alpha \circ u$. So, $M_{1}=\{[(\alpha, u)] \mid[(\beta, v)]=[(\alpha, u)]$ if $\beta \circ v=\alpha \circ u\}$. In a sense, we eliminated the artificial augmentation in the base space $\mathrm{N}$ by $D$. In other words, we reduced the parametrizations of minimal immersions to space $A$, since $M_{1} \simeq M \cap(A \times\{i d\})$.

Now, consider $C=\left\{c: D^{2} \rightarrow D^{2} \mid c\right.$ conformal $\left.c\right|_{\partial D^{2}}$ is class of $\left.C^{3}\right\}$. Now consider the action of $\mathrm{C}$ on $M_{1}$. Define for any $c \in C, \Phi_{c}: M_{1} \rightarrow M_{1}$ such that $\Phi_{c}([(\alpha, u)])=[(\alpha \circ c, u)]$, or by using the equivalence $M_{1} \simeq M \cap(A \times$ $\{i d\})$, then $\Phi_{c}((\alpha, i d))=(\alpha \circ c, i d)$. So, $M_{1} / C=\{([\alpha], i d) \mid \alpha \sim \beta$ if $\exists c \in C$, $\beta=\alpha \circ c\}$.

Now, if we can show that for any minimal plane $\Sigma \in M_{0}$, there exist unique $([\alpha], i d)$ with $\Sigma=\tilde{\alpha}\left(D^{2}\right)$, then we are done. Existence is true by section 2. Now, assume $\exists \alpha, \beta \in A$ such that $\Sigma=\tilde{\alpha}\left(D^{2}\right)=\tilde{\beta}\left(D^{2}\right)$. Then $\tilde{\beta}^{-1} \circ \tilde{\alpha}: D^{2} \rightarrow D^{2}$ is a conformal diffeomorphism of the disk. This implies $\beta^{-1} \circ \alpha \in C$, and $([\alpha], i d)=([\beta], i d)$, so the uniqueness follows.

Lemma 3.11. $[M O]$ Let a group $G$ acts freely on a manifold $X$, i.e. $\forall g \in$ $G-\{i d\}, \forall x \in X, g(x) \neq x$. Then the quotient space $X / G$ is a manifold.

Theorem 3.12. The space of minimal planes, $M_{0}$, is a manifold.

Proof. First, we will show that $M_{1}$ is a manifold, by showing the action of $\mathrm{D}$ on $\mathrm{M}$ is free. Let $v \in D$, and $\Psi_{v}((\alpha, u))=(\alpha, u)$. Then $\left(\alpha \circ v, v^{-1} \circ u\right)=$ $(\alpha, u)$. But, this implies $v^{-1} \circ u=u$ and so $v=i d$. The action is free, and by lemma $3.11, M_{1}$ is a manifold.

Now, if we can show the action of $C$ on $M_{1}$ is free, then we are done. Let $c \in C$, and $(\alpha, i d) \in((A \times\{i d\}) \cap M) \simeq M_{1}$, with $\Phi_{c}((\alpha, i d))=(\alpha, i d)$. Then $(\alpha \circ c, i d)=(\alpha, i d)$. But, this implies $\alpha \circ c=\alpha$, and $c=i d$. So, the action is free, and $M_{0}=M_{1} / C$ is a manifold.

\section{Generic Finiteness.}

In this section, our aim is to prove that for a generic $C^{3, \mu}$ simple Jordan curve $\Gamma \subset S_{\infty}^{2}\left(\mathbb{H}^{3}\right)$, there exist finitely many least area planes in $\mathbb{H}^{3}$ spanning $\Gamma$. From now on, we fix a $\mu>0$.

Let $M=\{(\alpha, u) \in N \mid \widetilde{\alpha \circ u}$ is minimal immersion $\}$ represents again the space of minimal immersions in $N$. We proved in previous section that $M$ 
is a submanifold of the product bundle $N=A \times D$ with $D \hookrightarrow A \times D \stackrel{\Pi}{\rightarrow} A$. Moreover, the projection map $\left.\Pi\right|_{M} \rightarrow A$ is Fredholm of index 0 .

Theorem 4.1. (Sard-Smale) $[S m]$ Let $f: X \rightarrow Y$ be a Fredholm map. Then the regular values of $f$ are almost all of $Y$, i.e except a set of first category.

Corollary 4.2. [Sm] Let $f: X \rightarrow Y$ be a Fredholm map. Then for any regular value $y \in Y, f^{-1}(y)$ is a submanifold of $X$ whose dimension is equal to index $(f)$ or empty.

Theorem 4.3. For almost all $\alpha \in A$, the set $\left(\left.\Pi\right|_{M}\right)^{-1}(\alpha)$ is a collection of isolated points in $M$.

Proof. Since $\left.\Pi\right|_{M}$ is onto by Theorem 3.9, $\left(\left.\Pi\right|_{M}\right)^{-1}(\alpha)$ is not empty for any $\alpha \in A$. Since $\left.\Pi\right|_{M}$ is $C^{2}$ Fredholm of index 0, by the corollary, $\left(\left.\Pi\right|_{M}\right)^{-1}(\alpha)$ is 0 dimensional submanifold of $\mathrm{M}$, for almost all $\alpha \in A$. The result follows.

Now, let $M^{\alpha}:=\left(\left.\Pi\right|_{M}\right)^{-1}(\alpha)=M \cap(\{\alpha\} \times D)$. So far we have shown that $M^{\alpha}$ is a collection of isolated points. If we can show it is also finite, then we are done.

Lemma 4.4. (Meeks-Yau) $[M Y]$ Let $M^{3}$ be a compact Riemannian threemanifold whose boundary is mean convex and let $\Gamma$ be a simple closed curve in $\partial M$ which is null-homotopic in $M$; then $\Gamma$ is bounded by a least area disk and any such least area disk is properly embedded.

Theorem 4.5. Let $\Gamma \subset S_{\infty}^{2}\left(\mathbb{H}^{3}\right)$ be a $C^{3, \mu}$ regular, simple Jordan curve. Then, any least area plane spanning $\Gamma$ is properly embedded.

Proof. By the boundary regularity results of Hardt and Lin in [HL], we can find a sufficiently large $N>0$ such that in Poincare Ball Model for $\mathbb{H}^{3}$, $\partial B_{N}(0) \cap \Sigma$ is a simple closed curve and $\Sigma-B_{N}(0)$ is properly embedded, indeed a graph over an annulus. Now, since $\Sigma \cap B_{N}(0)$ is also properly embedded by Lemma 4.4 as $B_{N}(0) \subset \mathbb{H}^{3}$ is convex, then result follows.

Lemma 4.6. Let $\Gamma \subset S_{\infty}^{2}\left(\mathbb{H}^{3}\right)$ be a $C^{3, \mu}$ curve. Then for any embedding $\varphi: D^{2} \rightarrow \mathbb{H}^{3}$ with $\varphi\left(\partial D^{2}\right)=\Gamma$ and $\varphi\left(D^{2}\right)$ least area plane, the $C^{3, \mu}$ norm of such minimal immersions are uniformly bounded by a constant depending on $\Gamma$, i.e. $\|\varphi\|_{C^{3, \mu}}<C(\Gamma)$. 
Proof. This follows from the results of Hardt and Lin in the articles [HL] and $[\mathrm{L}]$ about the boundary regularity of least area planes in hyperbolic space. In these papers, the authors proved that if $\Gamma \subset S_{\infty}^{2}\left(\mathbb{H}^{3}\right)$ be a $C^{3, \mu}$ curve, let $p \in \Gamma$, then there exist a neighborhood of point $N(p) \subset \overline{\mathbb{H}}^{3}$, such that for any minimal plane $\Sigma \subset \mathbb{H}^{3}$ spanning $\Gamma$, if $\phi$ parametrizes $\Sigma \cap N(p)$, then $\|\phi\|_{C^{3, \mu}}<C(p, \Gamma)$. Now, by using this local result, they conclude the boundary regularity of least area planes. Here, since interior regularity is already known, by using this local estimates and using simple transformations, we can reach a global estimate, such that any conformal harmonic parametrization of a least area plane spanning $C^{3, \mu}$ curve has $C^{3, \mu}$ norm uniformly bounded by constant depends only on curve and independent of the plane and its parametrization.

Theorem 4.7. Let $\Gamma \subset S_{\infty}^{2}\left(\mathbb{H}^{3}\right)$ be a $C^{3, \mu}$ be a generic curve as described above. Then there are finitely many least area planes spanning this curve.

Proof. Take a parametrization of $\Gamma$ in $A$, say $\alpha_{0}$. Then by previous sections, we know that all least area planes spanning $\Gamma$ have parametrizations in the form of $\widetilde{\alpha_{0} \circ u}$ with $\left(\alpha_{0}, u\right) \in M^{\alpha_{0}}$. So if we can show that $M^{\alpha_{0}}$ is finite, then the result follows. Now, since this set is bounded in $C^{3, \mu}$ topology by a constant $C(\Gamma)$ by Lemma 4.6 , it is compact in some $C^{3, \delta}$ topology where $0<\delta<\mu$. Now, if we can also show $M^{\alpha_{0}}$ is a collection of isolated points in $C^{3, \delta}$ topology, then this will imply $M^{\alpha_{0}}$ is finite.

Let $A_{\mu}=\left\{\alpha \in C^{3, \mu}\left(S^{1}, S^{2}\right) \mid \alpha\right.$ immersion $\}$, and let $M_{\mu} \subset A_{\mu} \times D$ be the submanifold corresponding minimal immersions. Similarly, define $A_{\delta}=\{\alpha \in$ $C^{3, \delta}\left(S^{1}, S^{2}\right) \mid \alpha$ immersion $\}$, and $M_{\delta}$. Now, by section $3, \Pi_{\mu}: M_{\mu} \rightarrow A_{\mu}$, and $\Pi_{\delta}: M_{\delta} \rightarrow A_{\delta}$ are Fredholm maps of index 0. Clearly, $M_{\mu} \subset M_{\delta}$ and $A_{\mu} \times D \subset A_{\delta} \times D$. Moreover, $\Pi_{\mu}=\left.\Pi_{\delta}\right|_{M_{\mu}}$. Now, we claim that if $\alpha \in A_{\mu} \subset A_{\delta}$ is a regular value for $\Pi_{\mu}$, then $\alpha$ is also a regular value for $\Pi_{\delta}$. This will imply $M^{\alpha_{0}}$ is a collection of isolated points in $C^{3, \delta}$ topology, by Lemma 4.3, and the result will follow.

Let's prove our claim: Let $\alpha \in A_{\mu}$ be a regular value for $\Pi_{\mu}$. Then for any $(\alpha, u) \in M_{\mu}, D \Pi_{\mu}: T_{(\alpha, u)} M_{\mu} \rightarrow T_{\alpha} A_{\mu}$ will be onto. Since $\Pi_{\mu}$ is Fredholm of index $0, D \Pi_{\mu}$ is an isomorphism. Now consider $D \Pi_{\delta}: T_{(\alpha, u)} M_{\delta} \rightarrow T_{\alpha} A_{\delta}$. The image of $D \Pi_{\delta}$ is closed as $D \Pi_{\delta}$ is Fredholm. Since $T_{\alpha} A_{\mu}$ is dense in $T_{\alpha} A_{\delta}$, and $\Pi_{\mu}=\left.\Pi_{\delta}\right|_{M_{\mu}}$, this would imply $D \Pi_{\delta}(\alpha, u)$ is also onto, and so $\alpha$ is also regular value for $\Pi_{\delta}$.

Then, $M^{\alpha_{0}}$ is collection of isolated points and compact in $C^{3, \delta}$ topology, and so it is finite. 


\section{Concluding Remarks.}

\subsection{Genericity.}

These results show generic finiteness for least area planes with $C^{3, \mu}$ smooth asymptotic boundary. But, one suspects that this is true in general with some smoothness condition on the boundary at infinity. In other words, it would be interesting to show that for a class of curves in $S_{\infty}^{2}\left(\mathbb{H}^{3}\right)$, say $C$, for any $c \in C$, the least area planes spanning $c$ is finite.

Another interesting question in the other direction might be the following: are there simple closed curves in $S_{\infty}^{2}\left(\mathbb{H}^{3}\right)$, which are spanned by infinitely many least area planes? In [A1], Anderson constructed least area planes for any simple closed curve in $S_{\infty}^{2}\left(\mathbb{H}^{3}\right)$. Maybe, one can develop his methods, and find different constructions giving different least area planes for same curve at infinity, and then get some curve at infinity spanned by infinitely many least area planes. In [G], Gabai gives a relatively different construction for laminations of least area planes for a given simple closed curve. By using that construction, it might be possible to answer positively the question.

\subsection{Regularity.}

In this paper, the $C^{3, \mu}$ condition can be relaxed to $C^{2, \mu}$ except Theorem 3.5, where we use $[\mathrm{ADN}]$ results. It might be interesting to know that what is the best class of curves to achieve such a generic finiteness result. On the other hand, we worked with Holder spaces in this paper. But, these methods can also work for Sobolev spaces instead of Holder spaces.

\subsection{Minimal vs. Least Area.}

We could also have a generic finiteness result for minimal planes if we can show that Lemma 4.6 is true for minimal planes, too. In that lemma, we needed least area assumption to apply [HL] and [L] in our situation. If one can show that same results are still true for minimal planes (mean curvature 0 planes), then this directly implies generic finiteness of minimal planes, by using our methods. 


\section{References.}

[ADN] S. Agmon, A. Douglis and L. Nirenberg, Estimates near the boundary for solutions of elliptic partial differential equaitons satisfying general boundary conditions. II., Commun. Pure Appl. Math. 17 (1965), 3592.

[A1] M. Anderson, Complete minimal varieties in hyperbolic space, Invent. Math. 69, 477-494 (1982).

[A2] M. Anderson, Complete minimal hypersurfaces in hyperbolicnmanifolds, Commnet. Math. Helv. 58, 264-290 (1983).

[BT] R. Bohme and F. Tomi, Zur Struktur der Lsungsmenge des Plateauproblems, Math. Z. 133, 1-29 (1973).

[CM] T. Colding and W.P. Minicozzi, Minimal surfaces, Courant Lecture Notes in Mathematics, 4, 1999.

[ER] J. Eells and A. Ratto, Harmonic maps and minimal immersions with symmetries, Ann. of Math. Studies, no. 130, Princeton Univ. Press, Princeton, NJ, 1993.

[ES] J. Eells and J.H. Sampson, Harmonic mappings of Riemannian manifolds, Amer. J. Math. 86, 109-159 (1964).

[G] D. Gabai, On the geometric and topological rigidity of hyperbolic 3manifolds, J. Amer. Math. Soc. 10, 37-74 (1997).

[HL] R. Hardt and F.H. Lin, Regularity at infinity for area minimizing hypersurfaces in hyperbolic space, Invent. Math. 88, 217-224 (1987).

[L] F.H. Lin, On the Dirichlet problem for the minimal graphs in hyperbolic space, Invent. Math. 96, 593-612 (1989).

[LRW] B.Lawruk, B. Rowley and J.T. Wloka, Boundary value problems for elliptic systems, Cambridge University Press, Cambridge, 1995.

[LT1] P. Li and L.F. Tam, Uniqueness and regularity of proper harmonic maps, Ann. of Math. (2),137, 167-201 (1993).

[LT2] P. Li and L.F. Tam, Uniqueness and regularity of proper harmonic maps. II, Indiana Univ. Math. J. 42, 591-635 (1993). 
[MO] J. Margalef and E. Outerelo, Differential topology, North-Holland Math. Studies, no. 173, North-Holland Publishing Co., Amsterdam, 1992.

[MY] W. Meeks and S.T. Yau, The classical Plateau problem and the topology of three manifolds, Topology 21, 409-442 (1982)

[Sm] S. Smale, An infinite dimensional version of Sard's Theorem, Amer. J. Math. 87, 861-866 (1965).

[To] Y. Tonegawa, Existence and regularity of constant mean curvature hypersurfaces in hyperbolic space, Math. Z. 221, 591-615 (1996).

[Tr] A.J. Tromba, On the number of simply connected minimal surfaces spanning a curve, Mem. Amer. Math. Soc., no.194 (1977).

[TT] F. Tomi and A.J. Tromba, Extreme curves bound embedded minimal surfaces of the type of the disc, Math. Z. 158, 137-145 (1978).

Department of Mathematics

Princeton University

PRINCETON, NJ 08544

baris@math . princeton . edu

Received August 8, 2003. 